02

\title{
Исследование молекулярного пучка Se масс-спектрометрическим методом с электронной ионизацией
}

\author{
() А.Н. Завилопуло, О.Б. Шпеник, А.М. Мылымко
}

Институт электронной фризики НАН Украины, 88017 Ужгород, Украина

e-mail: gzavil@gmail.com

(Поступило в Редакцию 26 февраля 2016 г. В окончательной редакции 6 мая 2016 г.)

Описаны методика и результаты масс-спектрометрических исследований образования положительных ионов в результате диссоциативной ионизации молекулярного пучка селена электронным ударом. Из кривых эффективности ионизации определены энергии появления фрагментных ионов. Исследована также динамика образования молекулярных ионов селена в интервале температур $420-500 \mathrm{~K}$. Впервые изучены энергетические зависимости эффективности образования однозарядных ионов $\mathrm{Se}_{n}^{+}$для $n=1-4$ и двузарядного иона селена в интервале от порога до $36 \mathrm{eV}$ и проанализированы обнаруженные особенности эффективных сечений ионизации.

DOI: 10.21883/JTF.2017.03.44235.1780

\section{Введение}

Селен является уникальным элементом, свойства которого зависят от условий его получения, а число атомов в молекулах селена зависит от температуры, что обусловливает существование аллотропических модификаций [1]. Селен изменяет свои свойства в зависимости от внешних условий: при атмосферном давлении существует несколько десятков модификаций селена. Наиболее стабилен серый селен $(\gamma$-Se, „металлический селен $\left.^{6}\right)$ с кристаллической решеткой его получают из других форм длительным нагреванием и медленным охлаждением расплава или паров селена, структура состоит из параллельных спиральных цепей. Кроме того, существуют три модификации красного кристаллического селена с моноклинной решеткой: $\alpha$-Se оранжево-красного цвета, $\beta$-Se - темно-красного цвета, $\gamma$-Se - красного цвета, который содержит кольцевые молекулы $\mathrm{Se}_{8}$. Известны еще две аллотропические модификации: красный аморфный селен и черный стекловидный селен [2]. Благодаря своим многогранным свойствам селен широко применяется в функциональной электронике и квантовой электронике [3], он входит в состав активных центров некоторых белков в форме аминокислоты селеноцистеина [4]. Селен относится к группе халькогенов вместе с химическими элементами VI группы Периодической системы Д.И. Менделеева: кислородом, серой, теллуром и полонием. Электронная оболочка атомов халькогенов имеет конфигурацию $n s^{2} n p^{4}$ и с увеличением атомного номера возрастают ковалентные и ионные радиусы атомов, уменьшается энергия ионизации атома и энергия диссоциации молекулы. Атомы халькогенов легко образовывают кластеры, например кластеры селена, характеризуются наличием цепочек и колец $\mathrm{Se}_{n}$ разных размеров $(n=1-25)$, которые могут находиться в газовой, твердой, жидкой и в аморфной фазах. Технология получения селена включает процесс осаждения исходного вещества из газовой фазы, поэтому знание компонентного состава его паров играет важную роль. Существует два основных источника информации об элементном составе селена в газовой фазе: данные термодинамических и масс-спектрометрических исследований, причем последние дают более полную и детальную информацию о наличии в парах селена тех или иных молекулярных компонент.

Известно [5,6], что природный селен состоит из пяти стабильных изотопов: ${ }^{74} \mathrm{Se}(0.87 \%$ по массе $),{ }^{76} \mathrm{Se}$ $(9.02 \%),{ }^{77} \mathrm{Se}(7.58 \%),{ }^{78} \mathrm{Se}(23.52 \%),{ }^{80} \mathrm{Se}(49.82 \%)$, a один $-{ }^{82} \mathrm{Se}(9.19 \%)$ испытывает двойной бета-распад с большим периодом полураспада. Кроме того, искусственно созданы еще 24 радиоактивных изотопа в диапазоне массовых чисел от 65 до 94. Первые шесть изотопов хорошо разделяются при масс-спектрометрических исследованиях. Молекулы селена с количеством атомов больше двух можно отнести к кластерным соединениям, а поскольку во многих аспектах кластеры представляют собой до сих пор недостаточно изученное состояние материи: нечто среднее между атомами и молекулами, с одной стороны, и твердого тела - с другой, то безусловно для выяснения природы этого состояния вещества необходима постановка новых экспериментов. Важную информацию о свойствах нейтральных атомных частиц, в том числе кластеров, можно получить, используя метод масс-спектрометрии электронного удара, путем изучения фрагментации исходного вещества в газовой фазе в процессе электронной ионизации.

Целью настоящей работы было получение новых данных о процессе образования ионов селена при электронной ионизации молекулярного пучка селена методом масс-спектрометрии при различных температурах испарения исходного вещества и энергиях бомбардирующих электронов. 


\section{Эксперимент}

Основой экспериментальной установки [7] являлся монопольный масс-спектрометр МX 7304A с разрешением по массе не хуже $\Delta M=1 \mathrm{u}$. Молекулярный пучок селена формировался с помощью многоканального источника эффузионного типа, который обеспечивал концентрацию молекул в области пересечения с электронным пучком в пределах $10^{10}-10^{11} \mathrm{~cm}^{-3}$. Усовершенствованный нами источник ионов с электронной ионизацией [8] работал в режиме стабилизации электронного тока и позволял получать пучки электронов с энергией от 5 до $90 \mathrm{eV}$ при токах $0.05-0.5 \mathrm{~mA}$ и разбросом по энергиям не хуже $\Delta E=0.3 \mathrm{eV}$. Калибровка шкалы масс выполнялась по изотопам атомов $\mathrm{Kr}$ и Хе, а шкала энергии электронов - по начальному участку сечения ионизации атома $\mathrm{Kr}$ и молекулы $\mathrm{N}_{2}$. Эксперимент проводился в два этапа [9]: на первом этапе исследовались масс-спектры при разных температурах вещества в источнике и энергиях ионизирующих электронов, а на втором - измерялись энергетические зависимости относительных сечений диссоциативной ионизации молекул селена в диапазоне энергий ионизирующих электронов от $5-30 \mathrm{eV}$.

\section{Результаты и их обсуждение}

\section{Macc-спектр}

Масс-спектрометрические исследования селена проведены во многих работах: электронным [9-14] и фотонным [15-17] ударами, а также с использованием техники сверхзвуковых пучков и молекулярной эпитаксии (MBE) [18-20]. Следует отметить, что в работах [21-23] проведены теоретические расчеты электронных состояний кластеров селена. Заметим, что в большинстве из перечисленных работ особое внимание уделено процессам фрагментации молекул селена в зависимости от температуры, которая существенным образом влияет на состав паров и соответственно на интенсивность образования молекулярных ионов.

При невысокой температуре $T<600 \mathrm{~K}$ селен в газовой фазе склонен образовывать цепи и кольца с числом атомов $n=2-8$, а при высокой температуре $T>800 \mathrm{~K}$ в сверхзвуковом пучке наблюдаются молекулы с числом атомов $n=40[19,20]$. Содержание различных фрагментов селена $\left(X_{n}\right)$ изучалось в работах [10-16]. Масс-спектрометрическим методом измерена зависимость $X_{n}=f(T)$ в диапазоне температур $450-1020 \mathrm{~K}$. На нижней границе этого диапазона основным фрагментом является $\mathrm{Se}_{6}$, а на верхней $-\mathrm{Se}_{2}$, т. е. при этих температурах интенсивно идет процесс термической деструкции тяжелых молекул $\mathrm{Se}_{n}$. В работе [14] исследован фракционный состав молекул при испарении в вакуум селена с открытой поверхности для двух модификаций: гексагонального селена и $\alpha$-Se. Установлено, что в обоих случаях преобладает $\mathrm{Se}_{6}$, а значительную

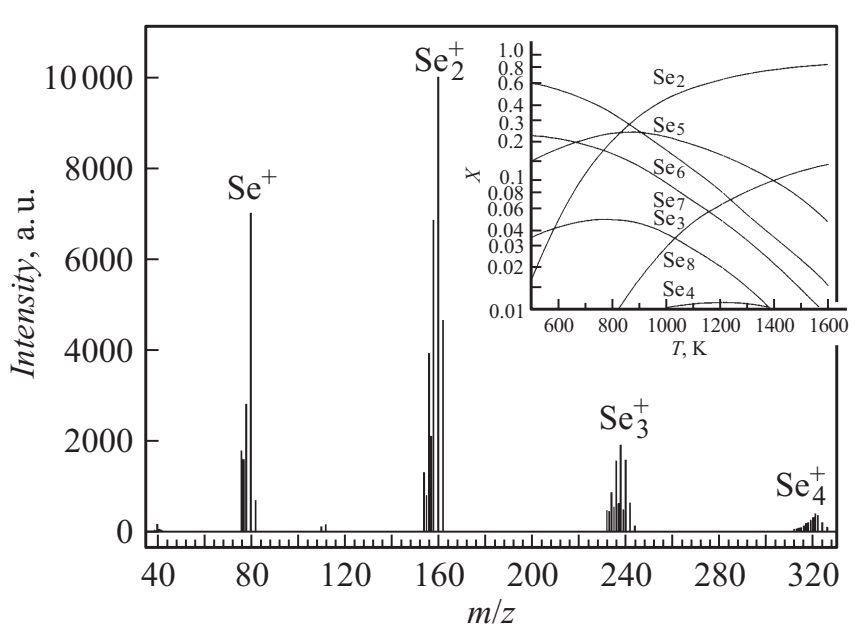

Рис. 1. Масс-спектр селена при $T=470 \mathrm{~K}$ и энергии ионизирующих электронов $U_{e}=70 \mathrm{eV}$. Вставка: зависимость фракционного состава молекулярной компоненты насыщенного пара селена от температуры [24].

высоту масс-пика $\mathrm{Se}_{2}$ авторы объясняют вкладом фрагментации молекул непосредственно в источнике ионов в результате электронной бомбардировки более тяжелых молекул $\mathrm{Se}_{n}$ (при энергии электронов $75 \mathrm{eV}$ ). Таким образом, в весьма широком диапазоне температур от самых низких $T=300$ до $1600 \mathrm{~K}$ состав насыщенного пара селена остается сложным и становится очевидным, что основной вклад в интенсивность пиков $\mathrm{Se}^{+}$и $\mathrm{Se}_{2}^{+}$в масс-спектрах вносится за счет процессов фрагментации молекулы селена $\mathrm{Se}_{6}$, концентрация которой в пучке при указанной температуре доминирует [14,24-26].

В ходе систематических исследований нами получено большое число масс-спектров селена при разной энергии налетающих электронов и различной температуре испарения вещества в диапазоне массовых чисел 1-350u. Наиболее характерный масс-спектр селена в диапазоне масс 35-350 u. показан на рис. 1, который получен при температуре источника молекул $T=470 \mathrm{~K}$ и энергии ионизирующих электронов $U_{e}=70 \mathrm{eV}$. Как видно из рисунка, в масс-спектре при этой температуре четко выделяется три группы пиков, максимальными по интенсивности в которых являются пики, соответствующие ионам атома $\mathrm{Se}^{+}(m / z=80)$ и молекул $\mathrm{Se}_{2}^{+}(m / z=160)$, $\mathrm{Se}_{3}^{+}(m / z=240)$ и $\mathrm{Se}_{4}^{+}(m / z=320)$, т. е. масс-пики, соответствующие стабильным атомным и молекулярным изотопам селена [6]. Такое распределение интенсивности ионных пиков молекул селена подтверждается приведенной из работы [24] зависимостью фракционного состава молекулярной компоненты насыщенного пара селена от температуры (вставка на рис. 1).

Заметим, что на приведенном масс-спектре (рис. 1) кроме основных масс-пиков селена наблюдаются также изотопные пики с разным соотношением интенсивности изотопного пика к основному. Например, величина изотопного пика атомарного иона селена ${ }^{78} \mathrm{Se}^{+}$составляет $39 \%$ от основного ${ }^{80} \mathrm{Se}^{+}$, причем такое же соотноше- 
ние сохраняется для пиков молекулярных ионов ${ }^{156} \mathrm{Se}_{2}^{+}$ и ${ }^{160} \mathrm{Se}_{2}^{+}$.

Обращает на себя внимание также наличие в массспектре небольшой группы пиков, максимальный из которых соответствует $m / z=40$, что свидетельствует об образовании двузарядного иона $\mathrm{Se}_{2}^{+}$в результате реакции

$$
\mathrm{Se}_{n}+e^{-} \rightarrow \mathrm{Se}^{++}+\mathrm{Se}_{n-1}+2 e^{-}
$$

\section{Температурные зависимости}

Исследования термодинамических особенностей селена в широком интервале температур проводилось во многих работах [10-16,24-26], в которых показано, что мольный состав паров и процесс деструкции сильно зависят от температуры испарения вещества и существенно влияют на количество атомов в кластеpax селена. Наибольшее число работ по исследованию температурных зависимостей образования ионных кластеров селена выполнено для температур выше $500 \mathrm{~K}$, что касается более низких температур, то таких работ значительно меньше. Поэтому нами были проведены детальные исследования масс-спектров молекулярного пучка селена в диапазоне температур $T=420-500 \mathrm{~K}$. Наши исследования однозначно подтвердили, что относительные интенсивности ионных пиков в масс-спектре существенно зависят от температуры испарения селена.

На рис. 2 приведены температурные зависимости для наиболее интенсивных пиков в масс-спектре, которые соответствуют ионам $\mathrm{Se}^{+}(m / z=80)$ и $\mathrm{Se}_{2}^{+}(m / z=160)$ и их изотопам: $m / z=78$ и $m / z=156$. Как видно, измеренные кривые имеют почти линейный рост с температурой, однако при $T=447 \mathrm{~K}$ на всех зависимостях имеется небольшой излом, глубина которого минимальная для изотопов (рис. 2, кривые 1,2 ).

Примечательно, что наблюдается различная скорость роста (угол наклона) кривых до излома и после него.

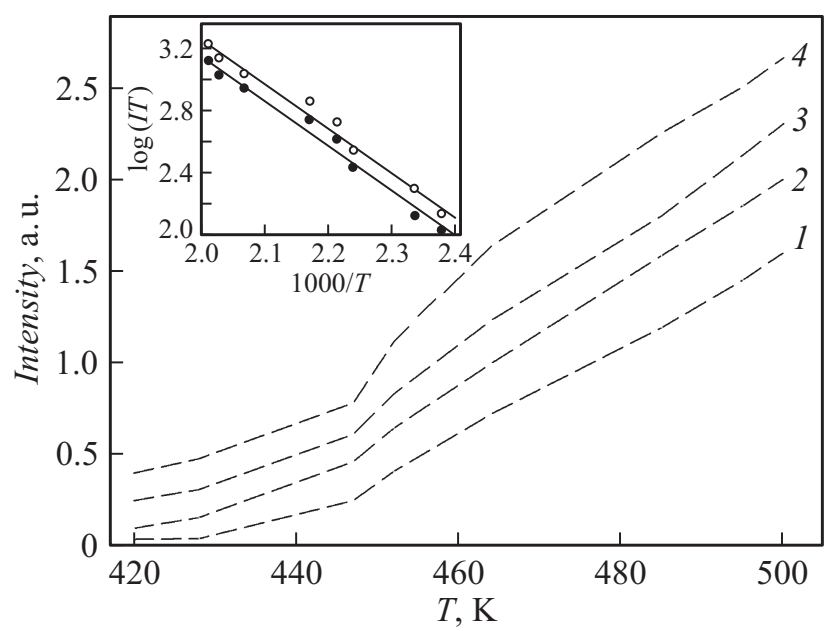

Рис. 2. Температурные зависимости для наиболее интенсивных пиков в масс-спектре, $m / z: 1-78,2-80,3-156$ и $4-160$. Вставка: зависимость температурных коэффициентов для $\mathrm{Se}_{2}^{+}-$о и $\mathrm{Se}^{+}-\bullet$.
По-видимому, после $T=447 \mathrm{~K}$ происходит заметное изменение состава пара (вставка на рис. 1) и интенсивность образования ионов $\mathrm{Se}^{+}, \mathrm{Se}_{2}^{+}$и их изотопов увеличивается (рис. 2). Кроме того, возникновение этой особенности на температурной зависимости, которая наиболее четко проявляется для $\mathrm{Se}_{2}^{+}$, может быть связано с термодинамическим изотопическим эффектом.

Поскольку давление паров исследуемого вещества пропорционально концентрации умноженной на температуру, т.е. давление в пучке селена будет пропорционально произведению величины ионного тока $(I)$ на температуру $(T)$. Следовательно, температурные коэффициенты можно получить из функциональной зависимости

$$
1 / T=f(\log I T) .
$$

На вставке рис. 2 показаны зависимости температурных коэффициентов для наиболее интенсивных пиков в масс-спектре, которые соответствуют ионам $\mathrm{Se}^{+}(m / z=80)$ и $\operatorname{Se}_{2}^{+}(m / z=160) .{ }^{1}$ Приведенные нами зависимости для температурных коэффициентов аналогичны полученным в [13], где сделано заключение о минимальном температурном коэффициенте для ионов $\mathrm{Se}_{6}^{+}$, который является родоначальником появления ионов $\mathrm{Se}^{+}$и $\mathrm{Se}_{2}^{+}$в результате диссоциативной ионизации. С другой стороны, исходя из зависимостей фракционного состава молекулярной компоненты насыщенного пара селена от температуры (вставка на рис. 1), можно приблизительно оценить процентный состав молекулярного пучка от общего числа молекул в условиях нашего эксперимента: $\mathrm{Se}_{2}^{+}-5, \mathrm{Se}_{5}^{+}-16$, $\mathrm{Se}_{6}^{+}-53, \mathrm{Se}_{7}^{+}-23$ и $\mathrm{Se}_{8}^{+}-4$. Таким образом, около 90\% всех молекул в пучке составляют кластеры $\mathrm{Se}_{5-7}$, тогда как количество $\mathrm{Se}_{3-4}$ очень мало. Заметим, что в интервале температур $T=470-450 \mathrm{~K}$ наблюдается отклонение от линейности зависимости температурных коэффициентов для всех ионов. Это, по-видимому, происходит в результате переходного процесса, связанного с изменением молекулярного состава, что приводит к увеличению интенсивности образования ионов $\mathrm{Se}_{2}^{+}$и $\mathrm{Se}^{+}$. Кроме того, возникновение этой особенности в изменении температурного коэффициента может быть связано еще и с термодинамическим изотопическим эффектом.

\section{Энергетические зависимости}

Настроив масс-спектрометр на выделение определенной массы, мы смогли измерять относительные сечения образования различных ионов селена в интервале энергий налетающих электронов от порога процесса до $40 \mathrm{eV}$. Отметим, что детальные исследования энергетических зависимостей образования ионов $\mathrm{Se}_{n}^{+}(n=1-9)$ от порога до $16 \mathrm{eV}$ проведено в недавней работе [27]. Нами же измерены энергетические зависимости образования ионов $\mathrm{Se}_{n}^{+}(n=1-4)$ от порога процесса до $36 \mathrm{eV}$.

\footnotetext{
1 Заметим, что подобные зависимости получены и для их основных изотопов: $m / z=78$ и $m / z=156$.
} 


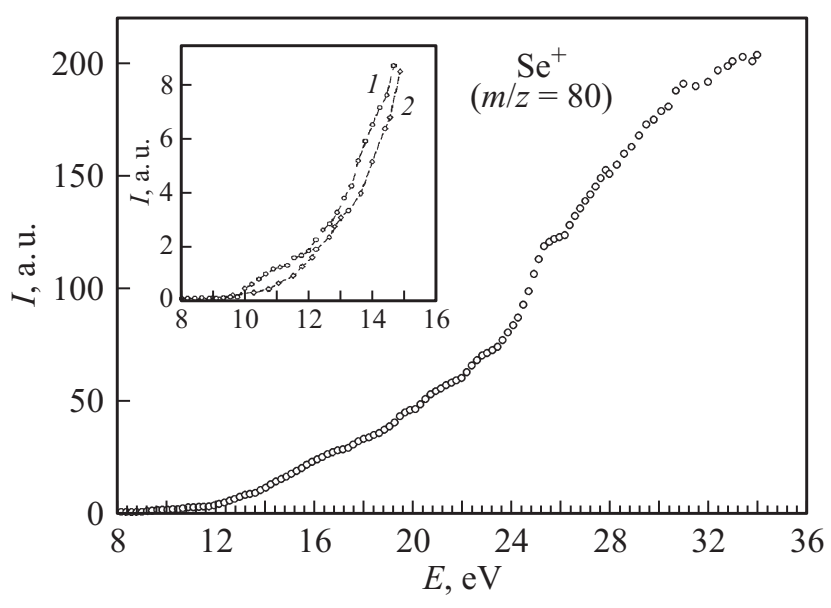

Pис. 3. Относительное сечение ионизации Se при $T=470 \mathrm{~K}$. Вставка: сравнение начальных участков относительного сечения ионизации $\mathrm{Se}$, полученных в настоящей работе -1 и в работе [27] -2 .

Полученная нами энергетическая зависимость образования ионов $\mathrm{Se}^{+}$представлена на рис. 3. На вставке рисунка приведено сравнение порогового участка этой зависимости с результатами работы [27]. Как видим, общий ход начальных участков кривых практически совпадает, однако, на нашей кривой вблизи порога наблюдается два небольших излома при энергиях 10.50 и $11.40 \mathrm{eV}$, которые могут быть связаны с потенциалами появления возбужденных состояний атома селена в результате диссоциативного возбуждения [28], либо в результате включения автоионизационного канала в процессе диссоциации при образовании данного иона. Таким образом, можно рассмотреть два наиболее вероятных канала образования монокатиона селена Se:

ионизащия атома селена электронами

$$
\mathrm{Se}+e^{-}=\mathrm{Se}^{+}+2 e^{-},
$$

диссоциативная ионизация молекул селена

$$
\mathrm{Se}_{n}^{+}+e^{-}=\mathrm{Se}^{+}+\mathrm{Se}_{n-1}+2 e^{-} .
$$

Как указывалось выше и в $[24,29]$, при испарении атомы селена в газообразном состоянии образуются в заметном количестве при температурах $>700 \mathrm{~K}$, поэтому в наших экспериментальных условиях $(T=420-500 \mathrm{~K})$ концентрация их ничтожно мала. Поэтому образование ионов $\mathrm{Se}^{+}$в этих условиях в основном идет по реакции (4) за счет процессов диссоциативной ионизации молекул $\mathrm{Se}_{5-7}$ при взаимодействии с электронами и в меньшей степени за счет молекул $\mathrm{Se}_{2}$, т.е. подобно аналогичным процессам, изученным нами для серы [30]. Ход кривой эффективности ионизации для иона $\mathrm{Se}^{+}$ отражает монотонный рост, однако имеется ряд особенностей при энергиях $E=19.20,20.40$ и $26.00 \mathrm{eV}$, которые свидетельствуют о включении дополнительного канала диссоциации с образованием данного иона.
Молекула $\mathrm{Se}_{2}$ является наиболее стабильной среди молекул халькогенов с малым числом атомов, что подтверждается максимальной интенсивностью иона $\mathrm{Se}_{2}^{+}$в масс-спектре. Ход энергетической зависимости образования этого иона (рис. 4) отличается от энергетической зависимости для ионов $\mathrm{Se}^{+}$: наблюдается довольно резкий подъем у порога и затем рост с наличием ряда максимумов при энергиях $15.00,16.20,21.00$ и $31.00 \mathrm{eV}$. На вставке рис. 4 (кривые 1,2) дано сравнение пороговых участков нашей энергетической зависимости с результатами работы [27], как видим, имеется хорошее согласие в поведении кривых. Источниками формирования иона $\mathrm{Se}_{2}^{+}$могут быть как процессы непосредственной ионизации молекул $\mathrm{Se}_{2}$, возникающих в процессе термической фрагментации, так и в результате диссоциативной ионизации молекул $\mathrm{Se}_{5-7}$ под действием электронного удара.

Нами впервые измерено также относительное сечение ионизации двузарядного иона селена $\mathrm{Se}^{++}(\mathrm{m} / \mathrm{z}=40)$, которое показано на рис. 5. Наиболее вероятным кана-

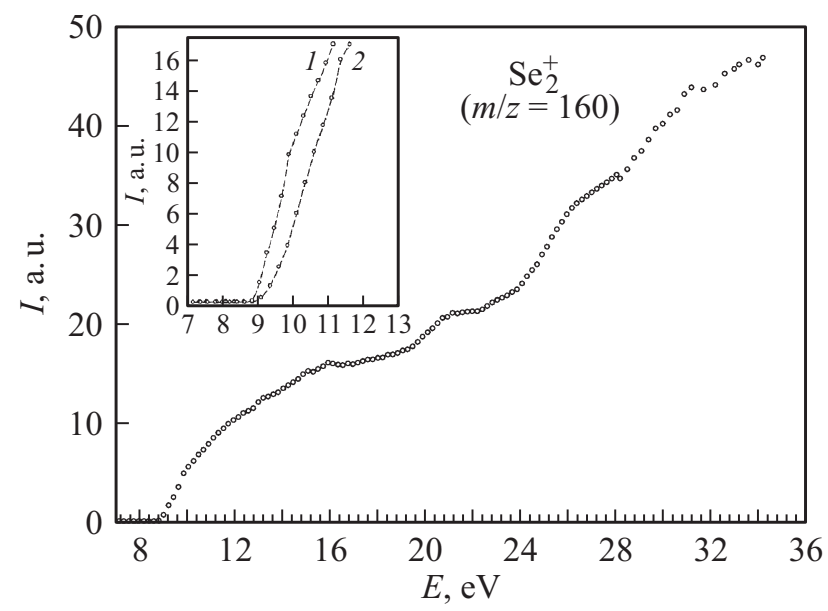

Рис. 4. Относительное сечение ионизации $\mathrm{Se}_{2}^{+}$при $T=470 \mathrm{~K}$. Вставка: сравнение начальных участков относительного сечения ионизации $\mathrm{Se}$, полученных в нашей работе -1 и в работе [27] -2 .

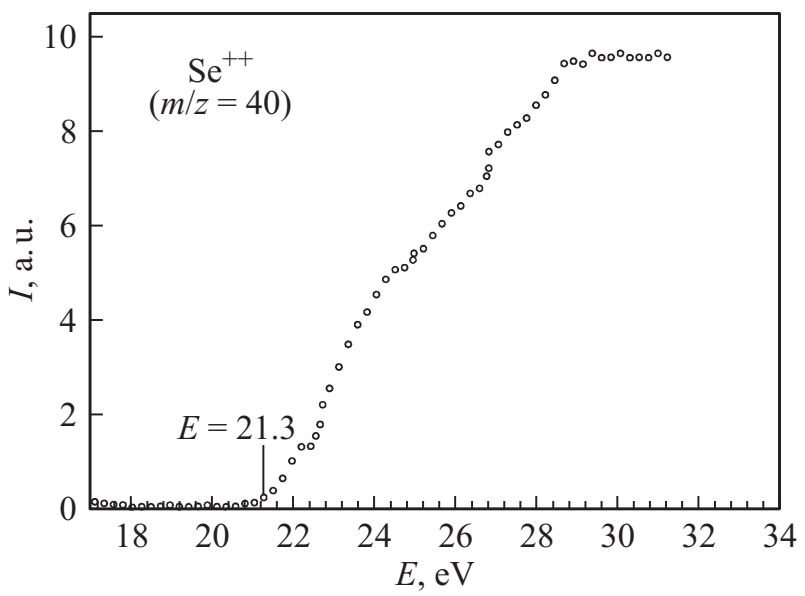

Pис. 5. Функция ионизации двузарядного иона селена. 
Относительные интенсивности ионных пиков и потенциалы их появления

\begin{tabular}{|c|c|c|c|c|c|c|c|c|c|c|c|}
\hline \multirow{3}{*}{ Ион } & \multirow{3}{*}{$\begin{array}{c}\text { Масса } \\
\text { иона, } \\
m / z\end{array}$} & \multirow{2}{*}{\multicolumn{4}{|c|}{$\begin{array}{c}\text { Относительная интенсивность } \\
\text { пиков, \% } \\
E=70 \mathrm{eV}\end{array}$}} & \multicolumn{6}{|c|}{ Потенциалы появления, eV } \\
\hline & & & & & & \multicolumn{4}{|c|}{ электронами } & \multicolumn{2}{|c|}{ фотонами } \\
\hline & & $\begin{array}{c}\text { наши } \\
\text { данные } \\
T=470 \mathrm{~K}\end{array}$ & $\begin{array}{c}{[11]} \\
T=600 \mathrm{~K}\end{array}$ & $T=544 \mathrm{~K}$ & $\begin{array}{c}\text { NIST } \\
{[31]}\end{array}$ & $\begin{array}{c}\text { наши } \\
\text { данные } \\
T=470 \mathrm{~K}\end{array}$ & $\begin{array}{c}{[11]} \\
T=600 \mathrm{~K}\end{array}$ & $\begin{array}{c}{[14]} \\
T=544 \mathrm{~K}\end{array}$ & $\begin{array}{c}{[27]} \\
T=480 \mathrm{~K}\end{array}$ & $\begin{array}{c}{[15]} \\
T=512 \mathrm{~K}\end{array}$ & $T=600 \mathrm{~K}$ \\
\hline $\mathrm{Se}^{+}$ & 80 & 70.1 & 19.5 & 34.9 & 69.0 & $9.81 \pm 0.25$ & $11.5 \pm 0.3$ & - & 9.4 & $9.75 \pm 0.05$ & 9.75 \\
\hline $\mathrm{Se}_{2}^{+}$ & 160 & 100 & 100 & 100 & 100 & $9.04 \pm 0.25$ & $9.8 \pm 0.3$ & $9.2 \pm 0.1$ & 9.4 & $8.84 \pm 0.05$ & 8.90 \\
\hline $\mathrm{Se}_{3}^{+}$ & 240 & 15.3 & 23.2 & 31.2 & 15.7 & $10.35 \pm 0.25$ & $11.7 \pm 0.1$ & $10.4 \pm 0.3$ & 10.2 & $9.58 \pm 0.05$ & 9.20 \\
\hline $\mathrm{Se}_{4}^{+}$ & 320 & 7.5 & 14.4 & 16.2 & 7.9 & $10.25 \pm 0.25$ & $10.1 \pm 0.1$ & $10.1 \pm 0.3$ & 10.8 & $9.14 \pm 0.05$ & 9.10 \\
\hline $\mathrm{Se}^{++}$ & 40 & 0.71 & - & - & 0.48 & $21.30 \pm 0.25$ & - & - & - & - & - \\
\hline
\end{tabular}

лом образования этого иона является реакция (1). Ход кривой эффективности ионизации двузарядного иона селена $\mathrm{Se}^{++}$, как видно из рисунка, начиная с энергии $E=21.2 \mathrm{eV}$, показывает монотонный рост с небольшими особенностями при энергиях $E=22.5,24.8$ и $26.8 \mathrm{eV}$ с последующим выходом на плато при $E>28.00 \mathrm{eV}$.

По пороговым участкам энергетических зависимостей образования наиболее интенсивных в масс-спектре ионов (рис. 1) методом наименьших квадратов [7] были определены энергии появления фрагментов, которые представлены в таблице. Там же приведены результаты относительных интенсивностей ионных пиков селена и дано сравнение наших данных с результатами базы данных NIST [31] и других авторов как электронным ударом $[11,14,27]$, так и фотонами $[15,16]$. Анализируя приведеннные в таблице данные, можно заключить, что в общем имеется удовлетворительное согласие между результатами, полученными разными методами. Основные расхождения как в относительной величине масс-пиков, так и в значениях потенциалов появления ионов разной кратности связаны с температурой паров селена, что напрямую свидетельствует о существовании аллотропических модификаций $\mathrm{Se}_{n}$. Интересно отметить, что, как отмечалось выше, пороговое поведение энергетических зависимостей образования ионов $\mathrm{Se}^{+}$и $\mathrm{Se}_{2}^{+}$, полученные в настоящей работе, практически совпадает (вставки на рис. 3,4$)$ с результатами, приведенными в [27], в которой также использовался метод масс-спектрометрии при одинаковых температурах испарения молекул селена. Однако имеется различие в энергии появления ионов $\mathrm{Se}^{+}$и $\mathrm{Se}_{2}^{+}$, если для $\mathrm{Se}^{+}$различие составляет $-0.4 \mathrm{eV}$, то для $\mathrm{Se}_{2}^{+}$оно равно $+0.4 \mathrm{eV}$ (см. таблицу).

\section{Заключение}

Впервые с помощью модернизированного монопольного масс-спектрометра проведены исследования молекулярного пучка селена масс-спектрометрическим методом: изучены масс-спектры, особенности фрагментации циклической молекулы селена в диапазоне температур $T=420-500 \mathrm{~K}$ и энергии ионизирующих электронов от порога процесса до $36 \mathrm{eV}$, измерены энергетические зависимости образования ионов $\mathrm{Se}_{n}^{+}$, установлены особенности испарения селена, изучены пороговые зависимости ионизации атомов и молекул селена, а также определены пороги появления ионов $\mathrm{Se}_{n}^{+}, n=1-4$, и двузарядного иона $\mathrm{Se}^{++}$. По результатам данных исследований можно сделать следующие выводы:

- состав молекулярного пучка содержит в основном многоатомные молекулы селена;

- на основе полученных масс-спектров изучены особенности фрагментации циклической молекулы селена, показано, что вероятность распада исходной молекулы зависит как от энергии ионизирующих электронов, так и от температуры испарения;

- в диапазоне температур $T=420-500 \mathrm{~K}$ исследованы температурные зависимости выхода образовавшихся ионов и установлено, что при $T=447 \mathrm{~K}$ наблюдается излом на кривой, связанный с заметным изменением фракционного состава молекулярного пучка;

- по пороговым участкам энергетических зависимостей эффективности образования однозарядных ионов $\mathrm{Se}^{+}-\mathrm{Se}_{3}^{+}$и двузарядного $\mathrm{Se}^{++}$найдены потенциалы их появления, которые удовлетворительно согласуются с данными других авторов.

\section{Список литературы}

[1] Weiser M.E., Holden N., Coplen T.B. et al. // Pure Appl. Chem. 2013. Vol. 85. N 5. P. 1047-1078.

[2] Mompean F.J., Perrone J. Chemical Thermodynamics / Ed. by F.J. Mompean, J. Perrone. Amsterdam: Elsevier, 2010. 894 p.

[3] Silfvast W.T., Klein M.B. // Appl. Phys. Lett. 1970. Vol. 17. N 9. P. 400-407.

[4] Johansson L., Gafvelin G., Amér E.S.J. // Biochim. Biophys. Acta. 2005. Vol. 1726. N 1. P. 1-13. DOI: 10.1016/j.bbagen.2005.05.010.

[5] NIST Standard Reference Database. http://www.webbook.nist.gov.

[6] Greenwood N., Earnshaw A. Chemistry of the Elements. 2nd ed. Oxford: Butterworth-Heinemann, 1997. P. 645-662.

[7] Завилопуло А.Н., Маркуи П.П., Шиеник О.Б. // ЖТФ. 2014. Т. 84. Вып. 7. С. 8-14. 
[8] Завилопуло А.Н., Миронеи, Е.А., Агафонова А.С. // ПТЭ. 2012. № 1. C. 73-79.

[9] Завилопуло А.Н., Шиеник О.Б., Микита М.И., Мылымко А.М. // Письма в ЖТФ. 2016. Т. 42. Вып. 8. С. 78-85.

[10] Brebrick R.F. // J. Phys. 1968. Vol. 48. N 12. P. 5741-5746.

[11] Yamdagni A., Porter R.F. // J. Electrochem. Soc. 1968. Vol. 115. P. 601-604.

[12] Grimley R.T., Grindstaff Q.G., De Mercurio T.A., Forsman J.A. // J. Phys. Chem. 1982. Vol. 86. P. 976-982.

[13] Fujisaki H., Westmore J.B., Tickner A.W. // Can. J. Chem. 1966. Vol. 44. P. 3063-3071.

[14] Berkowitz J., Chupka W.A. // J. Chem. Phys. 1966. Vol. 45. P. 4289-4294.

[15] Kooser K., Ha D.T., Itälä E. et al. // J. Chem. Phys. 2012. Vol. 137. P. 044304-9. doi: 10.1063/1.4737633

[16] Bréchignac C., Cahuzac Ph., Kébaïli N., Leygnier J. // J. Chem. Phys. 2000. Vol. 112. P. 10197-10 203; doi: $10.1063 / 1.481661$

[17] Andrew K. Hearley, Brian F.G. et al. // Inorg. Chim. Acta. 2002. Vol. 334. P. 105-112.

[18] Tribollet B., Benamar A., Rayane D. et al. // Z. Phys. D. 1993. Vol. 26. P. 352-354.

[19] Becker J., Rademann K., Hensel F. // Z. Phys. D. 1991. Vol. 19. P. 229-231.

[20] Minchev G.M., Eddrief M., Trendafilov L.M. et al. // Vacuum. 1996. Vol. 47. N 2. P. 157-165.

[21] Salaneck W.R., Iipari N.O., Paton A. et. al. // Phys. Rev. B. 1995. Vol. 52. N 3. P. 1524-1527.

[22] Li Z.Q., Yu J.Z., Ohno K. et al. // Phys. Rev. B. 1975. Vol. 12. N 4. P. $1493-1500$.

[23] Guptat G.P., Berrington K.A., Kingston A.E. // J. Phys. B. At. Mol. Opt. Phys. 1989. Vol. 22. P. 3289-3303.

[24] Смирнов Ю.М. // ТВТ. 2006. Т. 44. Вып. 5. С. 664-672.

[25] Berkowitz J., Chupka W.A. // J. Chem. Phys. 1968. Vol. 48. P. 5743-5744. doi: 10.1063/1.1668677

[26] Rau H. // J. Chem. Thermodyn. 1974. Vol. 6. N 4. P. 525-535.

[27] Viswanathan R., Balasubramanian R., Darwin D. et al. // J. Alloy Comp. 2014. Vol. 603. P. 75-85.

[28] Смирнов Ю.М. // ЖПС. 1991. Т. 55. № 2. С. 315-318.

[29] Справочник химика / Под ред. Б.П. Никольского. М.-Л.: Химия, 1982. Т. 1. С. 729.

[30] Завилопуло А.Н., Шиеник О.Б., Маркуш П.П. и др. // Письма в ЖТФ. 2014. Т. 40. Вып. 1. С. 29-36.

[31] NIST Standard Reference Database. http://www.webbook.nist.gov. 